\title{
The comparison of feasibility and safety on fiberoptic guided intubation under conscious sedation with remifentanil and propofol
}

\author{
Hae Mi Lee ${ }^{1}$, Jun Sakong ${ }^{2}$, and Dae-Lim Jee ${ }^{1}$ \\ Departments of ${ }^{1}$ Anesthesiology and Pain Medicine, ${ }^{2}$ Preventive Medicine and Public Health, College of Medicine, Yeungnam \\ University, Daegu, Korea
}

Background: Oropharyngeal manipulation is problematic when patients have a gag reflex. Sedation can suppress gag reflex, but can cause serious airway problems. We compared remifentanil (Group R) and propofol (Group P) in terms of cooperation and loss of gag reflex, while drugs were administered incrementally using target controlled infusion (TCI).

Methods: Fifty seven patients who required awake fiberoptic intubation were randomized to Group R or Group P. After measurement of baseline gag trigger point index (GTPI), TCI was set to effect-site concentration (Ce) of $1 \mathrm{ng} / \mathrm{ml}$ (Group $\mathrm{R}$ ) or $1 \mu \mathrm{g} / \mathrm{ml}$ (Group P), then titrated by 0.5 increment until GTPI score reached 0 . The incidence of drop-out and decreased cooperation, Ramsay sedation scale (RSS) and Ce at loss of GR, and complications were assessed.

Results: Seven patients were dropped out in Group P due to deep sedation and disobedient behavior, but none in Group $R(P=0.015)$. Gag reflex suppressed as RSS increased in both groups $(P<0.001)$, however, the incidence of elimination of gag reflex clustered at RSS 2 in Group $R(P<0.001)$, whereas it was evenly distributed in Group $P(P=0.20)$. The incidence of patients who were spontaneously roused (gag reflex elimination at RSS 1 and 2) were higher in Group R than in Group P (P $=0.002$ ).

Conclusions: Deep sedation and impaired cooperation were observed only in Group P and spontaneously roused patients were higher in Group R, suggesting that remifentanil is more suitable for cooperative elimination of GR. (Korean J Anesthesiol 2013; 65: 215-220)

Key Words: Gag reflex, Propofol, Remifentanil.

\footnotetext{
Received: January 4, 2013. Revised: March 7, 2013. Accepted: March 12, 2013.

Corresponding author: Dae-Lim Jee, M.D., Department of Anaesthesiology and Pain Medicine, Yeungnam University Hospital, 317-1, Daemyung-dong, Nam-gu, Daegu 705-035, Korea. Tel: 82-53-620-3364, Fax: 82-53-626-5275, E-mail: adjee@medical.yeungnam.ac.kr

(c) This is an open-access article distributed under the terms of the Creative Commons Attribution Non-Commercial License (http:// creativecommons.org/licenses/by-nc/3.0/), which permits unrestricted non-commercial use, distribution, and reproduction in any medium, provided the original work is properly cited.
} 


\section{Introduction}

Fiberoptic tracheal intubation, particularly orotracheal, is difficult without effective suppression of gagging and coughing. Gagging accounts for much of the procedural discomfort [1] and can occur even during topical anesthesia to the airway to obtund gagging and coughing for the procedure [2]. Elimination of gagging is vital for successful fiberoptic intubation as well as relieving patient discomfort.

Remifentanil or propofol used for sedation during fiberoptic intubation can eliminate gagging; elimination of gagging can facilitate topical anesthesia to the airway. Both drugs have different mechanisms responsible for elimination of gagging. Remifentanil is a potent opioid with less sedation, whereas propofol produces potent sedation without opioid action. These pharmacologic differences may lead to different patterns of side effects (impaired cooperation and undesirable sedation levels achieved, and their related adverse events) when used for sedation for elimination of gagging. In this study, we compared the risk of these side effects of remifentanil with propofol during the incremental target-controlled infusion (TCI) of remifentanil or propofol until the elimination of gagging, before spray-asyou-go topical anesthesia [3] for awake fiberoptic orotracheal intubation under conscious sedation.

\section{Materials and Methods}

After Institutional Ethics Committee approval (IRB No. 1036-15, approved at May 6th, 2010) and with written informed consent, 95 adult elective surgical patients (ASA physical status 1,2 ) who required fiberoptic orotracheal intubation were considered for the study (Fig. 1). Data were collected from May 2010 to May 2011 and patients with the following criteria were excluded: history of oropharyngeal surgery; use of drugs causing sedation or somnolence (hypnosis) or opioids; patients who cannot expose the posterior pharynx; a history of drug abuse and long-term use of benzodiazepines or tricyclic antidepressants.

\section{Gag assessment, drug preparation, blinding, and randomization}

Gagging was assessed using the gag trigger point index (GTPI) as modified from the original method [4]. The GTPI is an ordinal index in which the oropharyngeal regions are divided by anatomical landmarks and assigned a score (posterior pharynx with no motor response $=0$, posterior pharynx with motor response $=1$, between posterior faucial pillars and posterior pharyngeal wall $=2$, posterior faucial pillars $=$ 3 , between anterior and posterior faucial pillars $=4$, anterior faucial pillars $=5$, between second molars and anterior faucial

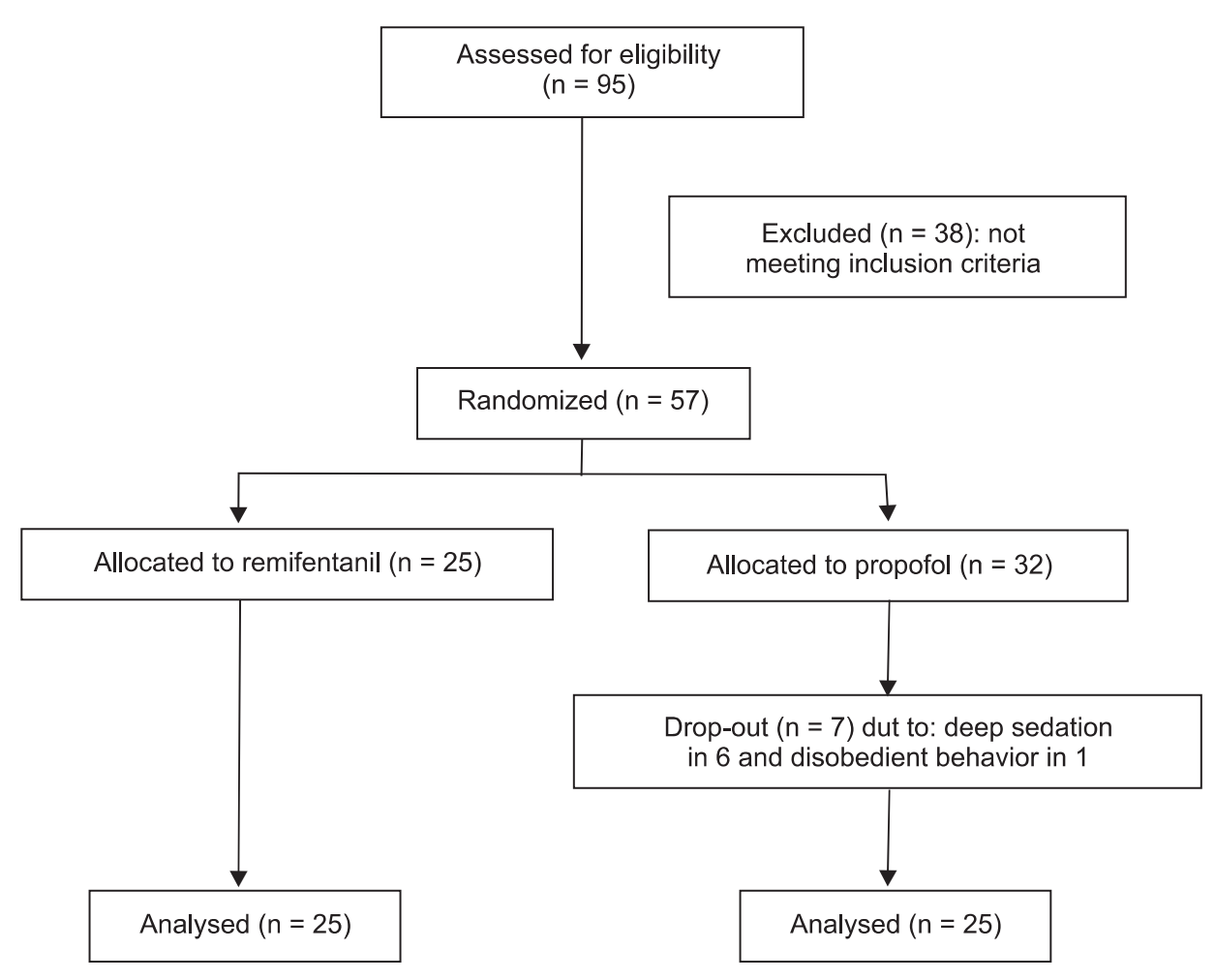

Fig. 1. Patient enrollment. 
pillars $=6$, second molars $=7$, internal cheek $=8$ ). We modified the severity rating scale system by removing the gag response sensor probe from the original method. Instead, patients were stimulated with cotton swabs and gagging was evaluated by observing contraction of uvula and soft palate, or the pharyngeal motor response, and inquiring whether the patient felt gagging or not. Stimulations to elicit gagging began from a high GTPI area (the internal cheek) to a low GTPI area (the pharyngeal wall): once a gag response was elicited, further posterior positions were not stimulated. Every patient was tested immediately before the study and the patient was excluded if he or she was free of gagging (Fig. 1). The patients were then randomized by using Excel software (version 2007, Microsoft Corp., Redmond, WA, USA) to administer either remifentanil (Ultiva $^{\circledR}$, GlaxoSmithKline, Parma, Italy, diluted with saline and given as a solution of $50 \mu \mathrm{g} / \mathrm{ml}$; remifentanil group) or propofol (Fresofol MCT 2\% solution, Fresenius Kabi, Graz, Austria; propofol group) via TCI system (Orchestra Base Primea ${ }^{\circledR}$, Fresenius-Vial, Brezins, France. Minto model for remifentanil and Marsh model for propofol). Two anesthesiologists carried out the study. One was responsible for performing elicitation and assessment of gagging, sedation scores, and patient's ability to cooperate, and the other for selecting the drug for TCI (infusion lines for both drugs were covered with linen to conceal which drug was infused), giving oxygen via a facemask and observing respiratory and circulatory parameters. The patient and the assessor were blinded to the selection of the drug and effect-site concentration (Ce) to be achieved.

\section{Study protocols}

On arrival in the operating theater, a $20 \mathrm{G}$ intravenous (IV) cannula was placed in an appropriate forearm vein for remifentanil or propofol TCI. The patient breathed spontaneously while receiving oxygen and air (total $4 \mathrm{~L} / \mathrm{min}, \mathrm{FiO}_{2} 0.4$ ) with face mask loosely applied throughout the study period. Standard monitor included three-lead electrocardiography (lead II), non-invasive arterial blood pressure, pulse oximetry $\left(\mathrm{SpO}_{2}\right)$, end-tidal $\mathrm{CO}_{2}\left(\mathrm{ETCO}_{2}\right)$, respiratory rate (RR), and heart rate (HR). The level of consciousness using modification of the Ramsay sedation scale (RSS) [5] was assessed and classified as deep (RSS $\geq 5$; unable to elicit and assess gagging), moderate (RSS 3-4; cooperation only with repeated command or sluggish obedience), minimal (RSS 2; calm but responsive to conversation) sedation, and alert (RSS 1). For convenience of assessment for rousability from sedation, consciousness (except for deep sedation) was further classified into spontaneously roused (RSS 1 and 2), in which the patient could understand and respond to health care providers' conversation, and non-spontaneously roused sedation (RSS 3 and 4), in which the patient was roused from sedation only in response to a command or physical stimulation. BIS was monitored in both groups, but the BIS data were only used for the propofol group. Remifentanil or propofol infusion was commenced at a target Ce of $1 \mathrm{ng} / \mathrm{ml}$ or $1 \mu \mathrm{g} / \mathrm{ml}$, respectively, and infusion rates were increased by 0.5 $\mathrm{ng} / \mathrm{ml}$ or $0.5 \mu \mathrm{g} / \mathrm{ml}$ increment, respectively, until the GTPI score reached 0 (disappearance of gagging) or the patient could not respond to the physician's command because of deep sedation or behavioral change. The changes of $\mathrm{BP}, \mathrm{SpO}_{2}, \mathrm{RR}, \mathrm{HR}$, RSS, BIS, and GTPI over the change of Ce were recorded before infusion (baseline) and then repeatedly $3 \mathrm{~min}$ (i.e., immediately before next elicitation of gagging) after each increment of $\mathrm{Ce}$ equilibrated with the plasma concentration. After completion of the study, topical anesthesia and awake fiberoptic intubation were performed, and total intravenous anesthesia using remifentanil and propofol was given for elective surgery.

The incidents of drug side effects were recorded. Impaired cooperation was defined as a state in which the patient opened his or her mouth only upon repeated orders (more than twice) for gagging assessment and finally could complete the course of elimination of gagging without drop-out (drop-out was defined as inability to maintain 'awake' state due to deep sedation or disobedient behavior). Adverse events were apnea, bradypnea, hypoxemia, hypotension, and bradycardia. Respiratory depression was defined as presence of apnea or bradypnea (RR $<9$ breaths/min). Apnea was defined as an absence of $\mathrm{ETCO}_{2}$ curve for more than 20 seconds on the monitoring display with no chest wall movement, but $\mathrm{ETCO}_{2}$ concentrations were not monitored because of loose mask fitting. Hypotension and bradycardia was defined as systolic blood pressure $<90 \mathrm{mmHg}$ or $\mathrm{HR}<50$ beats/min, respectively.

Incidence of drop-out and impaired cooperation were considered primary outcomes. Interim analysis showed that the sample size needed was 25 patients for each group to detect the difference between groups with a power of $80 \%$ and a type 1 error rate of 0.05 (rate of impaired cooperation was 0 in remifentanil group and 0.24 in propofol group). To compensate for the drop-out in propofol group (ratio of sample size of propofol group to remifentanil group $=1.25$ ), the sample size was estimated at 31 in propofol group.

\section{Statistics}

Results are presented as mean (standard deviation, SD), median (range), or number. Student's $t$ test was used to compare continuous variables between groups. Chi-square analysis, Fisher's exact test, Wilcoxon signed rank test, and Mann-Whitney $U$-test were used for non-continuous variables and continuous variables with non-normal distribution. The chi-square goodness-of-fit test was used to determine whether the frequency distribution 
according to sedation level were similar in each group. Generalized estimating equation (GEE) analysis was performed to assess the relationship between the change in $\mathrm{Ce}$ and the change in GTPI or RSS (and BIS in case of propofol group). P $<0.05$ was considered significant. All statistical analyses were conducted using SPSS ${ }^{\mathrm{TM}}$ statistical package 18 (SPSS Inc, an IBM Company, Chicago, IL, USA) and STATA ver. 6.0 (Stata Corp LP, College Station, TX, USA).

\section{Results}

Characteristics of patients who completed the study were shown in Table 1. Among the 95 patients who enrolled in this study, thirty eight patients $(40.0 \%)$ were free of gagging. Of the 57 patients who presented gagging, 50 completed the study: elimination of gagging was effective in both groups. The dropout rate was $21.9 \%$ (7/32) in patients using propofol (one dropped out because of disobedient behavior and six because of deep sedation), whereas none of the patients using remifentanil dropped out $(P=0.015)$. The incidence of deep sedation as a main cause of drop-out was $18.8 \%(6 / 32)$ in patients using propofol, compared with those using remifentanil $(0 \%)(\mathrm{P}=0.03)$.

For the assessment of gag reflex, 7 patients who manifested deep sedation or disobedient behavior were excluded, because

Table 1. Patient Characteristics Receiving Target-controlled Infusion for Elimination of Gagging

\begin{tabular}{lcc}
\hline & $\begin{array}{c}\text { Remifentanil } \\
(\mathrm{n}=25)\end{array}$ & $\begin{array}{c}\text { Propofol } \\
(\mathrm{n}=25)\end{array}$ \\
\hline Gender $(\mathrm{M} / \mathrm{F})$ & $11 / 14$ & $12 / 13$ \\
Age $(\mathrm{yr})$ & $47.6(11.9)$ & $44.4(12.2)$ \\
BMI $\left(\mathrm{kg} / \mathrm{m}^{2}\right)$ & $23.2(2.4)$ & $23.5(2.7)$ \\
HR $(\mathrm{beats} / \mathrm{min})$ & $70.2(13.1)$ & $72.8(12.1)$ \\
SAP $(\mathrm{mmHg})$ & $131.1(10.1)$ & $129.6(8.9)$ \\
RR $($ breaths/min) & $13.0(3.2)$ & $13.6(3.7)$ \\
Baseline GTPI & $3(1-6)$ & $3(1-5)$ \\
RSS at the time of elimination of gagging & $2(1-3)$ & $2(1-4)$ \\
Ce at the time of elimination of gagging & $2.4(0.8 \mathrm{ng} / \mathrm{ml})$ & $2.0(0.5 \mu \mathrm{g} / \mathrm{ml})$
\end{tabular}

Data are expressed as mean (SD), median (range), or numbers. BMI: body mass index, HR: heart rate, SAP: systolic arterial pressure, RR: respiratory rate, GTPI: gag trigger point index, RSS: Ramsay sedation scale, Ce: effectsite concentration. the final GTPI scores could not obtained from them. In both groups, baseline GTPI was lower in patients whose gagging was eliminated while alert (i.e., RSS $1, \mathrm{n}=7$ ) than while sedate (i.e., $\mathrm{RSS} \geq 2, \mathrm{n}=43)\{1(1-3)$ while alert vs. $3(1-6)$ while sedate, $\mathrm{P}=$ $0.009\}$.

The incidence of elimination of gagging clustered at the sedation level RSS 2 (23 out of 25 patients, 92\%) in remifentanil group $(\mathrm{P}<0.001)$, whereas it was distributed evenly irrespective of the levels of sedation ( $\mathrm{P}=0.20)$ (Table 2). The incidence of elimination of gagging at RSS 1 was not different between groups (4.0\% in remifentanil group vs. $24.0 \%$ in propofol group, $\mathrm{P}=0.098)$. The prevalence of patients who were spontaneously roused (i.e., RSS 1 and 2) from sedation was higher in remifentanil group than in propofol group at the time of elimination of gagging (96.0\% in remifentanil group vs. $60.0 \%$ in propofol group, $\mathrm{P}=0.002$ ).

In propofol group, BIS decreased at the time of elimination of gagging from the baseline during TCI in all patients [79 (68-98) at the time of elimination of gagging vs. baseline 96 (83-98), $\mathrm{P}$ $<0.001]$. BIS at the time of elimination of gagging in the patients whose gagging was eliminated while alert (i.e., RSS $1, n=6$ ) did not decrease significantly compared with their baseline BIS [92 (81-98) vs. baseline BIS 97 (96-98), $\mathrm{P}=0.07$ ].

A GEE analysis revealed that the change in GTPI and RSS (and BIS, in the case of propofol group) were associated with the change in Ce in both groups. GTPI decreased at an average rate of 0.43 for every increase of $0.5 \mathrm{ng} / \mathrm{ml}$ of Ce of remifentanil or 0.64 for $0.5 \mu \mathrm{g} / \mathrm{ml}$ of Ce of propofol $(\mathrm{P}<0.001)$. RSS increased at an average rate of 0.16 for every increase of $0.5 \mathrm{ng} / \mathrm{ml}$ of Ce of

Table 2. Frequency of Elimination of Gagging According to the Level of Sedation

\begin{tabular}{lcccccc}
\hline & Alert & & $\begin{array}{c}\text { Minimal } \\
\text { sedation }\end{array}$ & & \multicolumn{2}{c}{$\begin{array}{c}\text { Moderate } \\
\text { (conscious) sedation }\end{array}$} \\
\cline { 2 - 3 } \cline { 5 - 6 } \cline { 5 - 6 } & RSS 1 & & RSS 2 & & RSS 3 & RSS 4 \\
\hline Remifentanil (n) & 1 & & 23 & & - \\
Propofol (n) & 6 & & 9 & & 8 & 2 \\
\hline
\end{tabular}

Data are expressed as numbers. The patients were distributed evenly according to RSS in propofol group $(\mathrm{P}=0.20)$ whereas it was not in remifentanil group $(\mathrm{P}<0.001)$. RSS: Ramsay sedation scale.

Table 3. Average Changes in GTPI, RSS, and BIS Associated with Increase of Effect-site Concentration of Remifentanil and Propofol

\begin{tabular}{|c|c|c|c|c|c|c|}
\hline & \multicolumn{3}{|c|}{$\begin{array}{c}\text { Remifentanil } \\
(0.5 \mathrm{ng} / \mathrm{ml})\end{array}$} & \multicolumn{3}{|c|}{$\begin{array}{l}\text { Propofol } \\
(0.5 \mu \mathrm{g} / \mathrm{ml})\end{array}$} \\
\hline & Coefficient & SE & $95 \% \mathrm{CI}$ & Coefficient & SE & $95 \%$ CI \\
\hline GTPI & -0.43 & 0.07 & $-0.29--0.57$ & -0.64 & 0.09 & $-0.82--0.46$ \\
\hline RSS & 0.16 & 0.09 & $0.10-0.21$ & 0.56 & 0.06 & $0.44-0.68$ \\
\hline BIS & - & - & - & -4.30 & 0.61 & $-5.48^{--3.11}$ \\
\hline
\end{tabular}

GTPI: gag trigger point index, RSS: Ramsay sedation scale, BIS: bispectral index. 
Table 4. Frequency of Adverse Events during Elimination of Gagging

\begin{tabular}{lcc}
\hline & Remifentanil $(\mathrm{n}=25)$ & Propofol $(\mathrm{n}=25)$ \\
\hline Hypotension $(<90 \mathrm{mmHg})$ & 0 & 0 \\
Bradycardia $(<50$ beats $/ \mathrm{min})$ & 2 & 1 \\
Respiratory depression & 5 & 6 \\
Apnea & 1 & 5 \\
Bradypnea $(<9$ breaths/min $)$ & 5 & 5 \\
Hypoxia $\left(\mathrm{SpO}_{2}<90 \%\right)$ & 0 & 0
\end{tabular}

Data are expressed as numbers. There are no significant differences between groups.

remifentanil or 0.56 for $0.5 \mu \mathrm{g} / \mathrm{ml}$ of Ce of propofol $(\mathrm{P}<0.001)$. BIS decreased at an average rate of 4.30 for every increase of 0.5 $\mu \mathrm{g} / \mathrm{ml}$ of Ce of propofol $(\mathrm{P}<0.001$, Table 3$)$.

Six patients in the propofol group showed impaired cooperation compared with none in remifentanil group $(\mathrm{P}=0.02)$. The incidence of adverse events was not statistically different between groups (Table 4). Bradypnea (the lowest RR was 6 breaths/min) and apnea did not require treatment. The patients recovered ventilation spontaneously after a bout of bradypnea or apnea, or by verbal commands for deep breaths. Bradycardia was closely monitored (the slowest HR is approximately 47-49).

\section{Discussion}

In this study, patients using propofol TCI displayed a high incidence of drop-out, which was not observed in patients using remifentanil TCI. The incidence of patients who were spontaneously roused from sedation was lower in propofol group and impaired cooperation was also observed only in patients using propofol. Gagging was suppressed dose-dependently (precisely, Ce-dependently) in both groups, although the differences were not significant in the risk of adverse events at the time of elimination of gagging between two groups. These findings suggest that propofol may present the potential for deep sedation compared with the use of remifentanil in our setting.

Deep sedation, disobedient behaviors, and/or impaired cooperation may be undesirable for patients with whom sprayas-you-go technique should be performed, or whose airway should not be compromised. Gagging was eliminated by sacrificing cooperation in some patients using propofol, which could disturb the patient and the clinician. It was interesting to note that deep sedation with consequent failure to complete the study was found only in patients using propofol. We expected that propofol TCI could help prevent deep sedation, however, patients using propofol showed a high rate of deep sedation. We speculate that the potent sedative property of propofol predominates before elimination of gagging. Thus, remifentanil may be more suitable for elimination of gagging in patients who require awake fiberoptic intubation.

Sedation with propofol has been thought to suppress gagging, although the effect of sedation on elimination of gagging has not been elucidated. Deep sedation and general anesthesia can eliminate gagging completely without cooperation, whereas minimal to moderate sedation may cause discomfort in awake patients, but with cooperation. Surprisingly, propofol eliminated gagging without causing sedation in $24 \%$ of the patients in the present study. Sedation scales (RSS and BIS) confirmed that their consciousness was not depressed. Moreover, patients had the diverse levels of sedation at the time of elimination of gagging and GEE analysis identified that GTPI decreased Cedependently. These results imply that elimination of gagging may not be related to sedation. However, it was difficult to separate the dose effect from the sedation effect on elimination of gagging, because increasing Ce produced increasing sedation.

Propofol given at subhypnotic dose is involved in elimination of postoperative nausea and vomiting (PONV) [6] and it is unknown whether these doses of propofol can also eliminate gagging. Interestingly, our results found lower GTPI in patients whose gagging was eliminated at RSS 1(i.e., without sedation) than in patients at RSS $\geq 2$ (i.e., with sedation). This finding suggests that patients with lower GTPI require lower Ce. On the other hand, elimination of gagging at a subhypnotic dose seemed to be very low in the dental literature, and we found only one case reported in which gagging in response to posterior oral stimulation, such as insertion of a dental mirror, was eliminated by propofol at a subhypnotic dose (a bolus injection of $0.2 \mathrm{mg} / \mathrm{kg}$ followed by the continuous infusion of $4 \mathrm{mg} / \mathrm{kg} / \mathrm{h}$ ) [7]. One probable explanation why elimination of gagging at a subhypnotic dose was unexpectedly high in our study might be that the patients were continuously exposed to various stimuli, such as regular BP measurements, stimulation to oral cavity and pharynx, and verbal communications, perhaps keeping the patients alert and agitated even during drug infusion, thus masking the sedating effect of the drugs despite increased Ce.

Gagging was previously reported to be absent in $43 \%$ of the elderly subjects and $26 \%$ of the young subjects, averaging $37 \%$ [8], not much different from our result. We do not know the clinical significance of this absence of gagging during the airway endoscopy. Our personal experience is that gagging seemed to be elicited when the fiberoptic bronchoscope was advanced deeply down to the hypopharynx in patients whose gagging was absent on the stimulation to the pharynx.

Some researchers proposed that gagging and nausea represent different stages of a process that might culminate in full blown vomiting [9]. Gagging is assumed to be controlled from the vomiting center [10]. Acupuncture, which covers the spectrum of gagging from the mild end of nausea to the severe end that culminates in vomiting [10], is reported to reduce gagging, 
nausea and/or emesis without sedation, probably by both a peripheral and central effect involving neurochemical substances such as serotonin and $\beta$-endorphin [10]. These neurochemical substances might partly explain the role of propofol (serotonin) and remifentanil ( $\beta$-endorphin) in eliminating gagging [10]. Furthermore, the antiemetic action of propofol can be attributed to interaction of propofol with dopaminergic, limbic and serotonergic system [11-13], suggesting that the antiemetic action of propofol might be related to elimination of gagging. Moreover, opioid receptors within the blood-brain barrier may mediate an endogenous system of anti-emesis with high lipid solubility opioids [14]. These reports imply that remifentanil eliminates gagging partly in this way. However, it is interesting that remifentanil is not effective for PONV and sedation is purported to be effective for gagging, but not for PONV. The reason remifentanil and sedation are not effective for PONV should be explainable if we accept that gagging and nausea represent different stages of a process [9]. Obviously, remifentanil may blunt noxious stimulation (such as gag stimulation) via activation of $\mu$-opioid receptors [14].

There were certain limitations in this study. One of the methodological considerations was the exclusion of 7 patients who showed deep sedation or disobedient behavior for the analysis of gag reflex. It was inevitable because Ce and RSS score of GTPI 0 was mandatory to assessment of gag reflex. However, we could not get those data from these patients, because they were not allowed to open their mouths. Therefore, gag reflex was analyzed in compensated 50 patients whose final GTPI reached to 0 . However, the results may not differ from those of data including 7 dropped-out cases; rather, it may be more significant.

One of our concerns in this study was to identify the difference of impaired cooperation between the two drugs in the course of elimination of gagging. The sample size was not small for that purpose. However, the sample size was not large enough to estimate the incidence of adverse events between groups. Thus, we could not determine which drug is safer, although we speculate that propofol has a propensity for pharmacologic property-related problems, such as deep sedation and impaired cooperation.

In summary, impaired cooperation and deep sedation with consequent drop-out were observed only in patients using propofol. The incidence of patients who were spontaneously roused from sedation was lower in patients using propofol, with no difference in adverse events between patients using remifentanil and propofol, suggesting that remifentanil may be more suitable for elimination of gagging in patients for awake fiberoptic intubation. Because gagging was eliminated in the diverse levels of sedation, we postulate that sedation might not be a unique mechanism responsible for elimination of gagging. Remifentanil and propofol may eliminate gagging in a dose-dependent rather than sedation-dependent manner.

\section{References}

1. Tsui BC, Dillane D, Yee MS. Patient-controlled oral airway insertion to facilitate awake fibreoptic intubation. Can J Anaesth 2008; 55: 194-5.

2. Farhadi A, Fields JZ, Hoseini SH. The assessment of esophagogastroduodenoscopy tolerance a prospective study of 300 cases. Diagn Ther Endosc 2001; 7: 141-7.

3. Williams KA, Barker GL, Harwood RJ, Woodall NM. Combined nebulization and spray-as-you-go topical local anaesthesia of the airway. Br J Anaesth 2005; 95: 549-53.

4. Scarborough D, Bailey-Van Kuren M, Hughes M. Altering the gag reflex via a palm pressure point. J Am Dent Assoc 2008; 139: $1365-72$.

5. Ramsay MA, Savege TM, Simpson BR, Goodwin R. Controlled sedation with alphaxalone-alphadolone. Br Med J 1974; 2: 656-9.

6. Borgeat A, Wilder-Smith OH, Saiah M, Rifat K. Subhypnotic doses of propofol possess direct antiemetic properties. Anesth Analg 1992; 74: 539-41.

7. Tomioka S, Uchida D, Eguchi S, Nakajo N. Elimination of hypersensitive gagging reaction to dentistry by propofol at subhypnotic doses. Oral Dis 1998; 4: 279-80.

8. Davies AE, Kidd D, Stone SP, MacMahon J. Pharyngeal sensation and gag reflex in healthy subjects. Lancet 1995; 345: 487-8.

9. Chate RA. Study limitations. Br Dent J 2007; 202: 705.

10. Rosted P, Bundgaard M, Fiske J, Pedersen AM. The use of acupuncture in controlling the gag reflex in patients requiring an upper alginate impression: an audit. Br Dent J 2006; 201: 721-5.

11. Vasileiou I, Xanthos T, Koudouna E, Perrea D, Klonaris C, Katsargyris A, et al. Propofol: a review of its non-anaesthetic effects. Eur J Pharmacol 2009; 605: 1-8.

12. Cechetto DF, Diab T, Gibson CJ, Gelb AW. The effects of propofol in the area postrema of rats. Anesth Analg 2001; 92: 934-42.

13. Funahashi M, Mitoh Y, Matsuo R. The sensitivity of hyperpolarization-activated cation current (Ih) to propofol in rat area postrema neurons. Brain Res 2004; 1015: 198-201.

14. Johnston KD. The potential for mu-opioid receptor agonists to be anti-emetic in humans: a review of clinical data. Acta Anaesthesiol Scand 2010; 54: 132-40. 